\title{
Long non-coding RNA HNF1A-AS1 promotes cell viability and migration in human bladder cancer
}

\author{
ZHIHONG FENG and BAOLONG WANG \\ Urology Department, Tianjin Medical University General Hospital, Tianjin 300052, P.R. China
}

Received October 19, 2015; Accepted January 22, 2018

DOI: $10.3892 / \mathrm{ol} .2018 .7878$

\begin{abstract}
Bladder cancer is among the most frequent types of genitourinary malignancies and results in high morbidity and mortality. Despite considerable progress in methods of bladder cancer diagnosis and treatment, the detailed underlying molecular mechanisms of bladder cancer remain unclear, and the prognosis of patients remains poor. In the present study, the role of long non-coding (lnc)RNA hepatocyte nuclear factor 1A (HNF1A)-antisense RNA (AS)1 in bladder cancer progression was examined in vitro. HNF1A-AS1 was overexpressed in clinical bladder cancer tissues and cultured bladder cancer cells. Specific short hairpin RNAs against HNF1A-AS1 knocked down the expression of HNF1A-AS1, and thus suppressed the viability and migration/invasion abilities of the cells. Additionally, the depletion of HNF1A-AS1 in bladder cancer T24 and 5637 cell lines also induced cell accumulation in $G_{0} / G_{1}$ phase with the cell cycle analysis. Overall, these data suggest that lncRNA HNF1A-AS1 may be a potential regulator of bladder cancer tumorigenesis, and provide novel insight into the diagnosis and treatment of bladder cancer.
\end{abstract}

\section{Introduction}

Bladder cancer (BC) has the second most frequent incidence of cancers of the genitourinary tract worldwide (1). In 2012 alone, 430,000 incident patients with BC and 165,000 BC-associated mortalities were identified (1). Although advances in treatment have been made in previous decades, the recurrence rate of $\mathrm{BC}$ is between 15 and $90 \%$ within 5 years, and the 5-year-survival-rate is $\sim 75 \%$ (2-5). In total, $\sim 15 \%$ of patients with papillary BC develop muscle-invasive and metastatic cancer (3). In these patients, long-term medical care is required, with inevitably high personnel and socioeconomic cost (6). Therefore, it is important to identify novel clues to diagnose and treat $\mathrm{BC}$.

Correspondence to: Professor Baolong Wang, Urology Department, Tianjin Medical University General Hospital, 154 Anshanxi Road, Heping District, Tianjin 300052, P.R. China E-mail: baolongwang789@sina.com

Key words: long non-coding RNA, cell viability, hepatocyte nuclear factor 1A-antisense RNA 1, bladder cancer
With the identification of long non-coding RNAs (lncRNAs), it has been demonstrated that certain lncRNAs may serve important roles in BC (5,7-10). LncRNAs are a category of non-coding RNAs, containing $>200$ nucleotides with limited translation potential. Unlike other non-coding RNAs, including microRNAs, the underlying molecular mechanisms of different lncRNAs involved in human diseases remain largely unknown. To date, lncRNAs have been demonstrated to participate in multiple intracellular and extracellular activities, including gene transcription, mRNA splicing and tumorigenesis (11-15). For example, the dysregulation of lncRNAs has been demonstrated in different types of cancer including hepatocellular carcinoma-upregulated lncRNA in hepatocellular carcinoma (16), metastasis-associated lung adenocarcinoma transcript 1 in lung cancer (17) and urothelial cancer-associated 1 and ZEB2 natural antisense transcript in BC $(18,19)$.

Unfortunately, despite the increasing implications of lncRNAs in bladder tumorigenesis, no lncRNA has been widely recognized as a specific biomarker for the progression or prognosis of $\mathrm{BC}$. This fact, to a certain extent, encourages focus on novel lncRNAs in BC. Hepatocyte nuclear factor 1A (HNF1A)-antisense RNA (AS)1 is a newly identified lncRNA. Since its initial identification, HNF1A-AS1 has been demonstrated to serve a critical role in human tumorigenesis, including esophageal adenocarcinoma (20) and lung adenocarcinoma (21) The expression of HNF1A-AS1 was upregulated in esophageal and lung adenocarcinoma cells (21). Functionally, HNF1A-AS1 promotes cell viability and metastasis in these cell types $(20,21)$, indicating that HNF1A-AS1 may promote cancer progression. However, whether HNF1A-AS1 exerts a robust cancer-promotion effect in BC cells has not been extensively studied.

In the present study, the expression of HNF1A-AS1 in patients with $\mathrm{BC}$ and cultured cells was examined using the quantitative polymerase chain reaction (qPCR). The specific role of HNF1A-AS1 in BC cell viability and metastasis was investigated using cell viability, colony formation, cell migration and invasion assays. Hopefully, these results may lead to the identification of novel methods to diagnose and treat patients with $\mathrm{BC}$ in clinical settings.

\section{Materials and methods}

Human samples. Between January 2011 and December 2014, a total of 30 patients with $\mathrm{BC}$ who were admitted to Tianjin 
Medical University General Hospital (Tianjin, China) and underwent a surgical resection were included. Cancerous tissues and their adjacent non-cancerous tissues were obtained from each patient, and used for the subsequent analysis. All patients provided full written informed consent to participate in the present study, and the study protocol was approved by the Ethics Committee of Tianjin Medical University.

Cell lines. A total of four human $\mathrm{BC}$ cell lines, including T24, J82, UMUC3 and 5637, and a normal bladder cell line SV-HUC-1 were purchased from the Cell Bank of the Chinese Academy of Sciences (Shanghai, China). Cells were cultured in the Dulbecco's modified Eagle's medium (DMEM; Gibco; Thermo Fisher Scientific, Inc., Waltham, MA, USA) supplied with $10 \%$ fetal bovine serum (FBS; Gibco; Thermo Fisher Scientific, Inc.) without antibiotics. Cells were maintained at $37^{\circ} \mathrm{C}$ in an incubator with a humidified atmosphere containing $5 \% \mathrm{CO}_{2}$. Medium was refreshed every other day, except where otherwise stated.

$R N A$ isolation and $q P C R$. To extract the total RNA from human tissues and cultured cells, TRIzol reagent (Thermo Fisher Scientific, Inc.) was used according to the manufacturer's protocol. A $1 \mu \mathrm{g}$ amount of total RNA from each sample was then reverse-transcribed into cDNA $\left(37^{\circ} \mathrm{C}\right.$ for $15 \mathrm{~min}$ and $85^{\circ} \mathrm{C}$ for $5 \mathrm{sec}$ ) using a PrimeScript RT Master Mix Perfect Real Time kit (Takara Biotechnology Co., Ltd., Dalian, China). Subsequently, qPCR was performed using the SYBR Premix Ex Taq kit (Takara Biotechnology Co., Ltd.) with the following primers: HNF1A-AS1 forward, 5'-TCAAGAAATGGTGGCTAT-3' and reverse, 5'-GCTCTG AGACTGGCTGAA-3'; GAPDH forward, 5'-GTGGACATC CGCAAAGAC-3' and reverse, 5'-AAAGGGTGTAACGCA ACTA-3'. The sequence of Thermocycling was as follows: $95^{\circ} \mathrm{C}$ for 2 min followed by 40 cycles of $95^{\circ} \mathrm{C}$ for $15 \mathrm{sec}$ and $60^{\circ} \mathrm{C} 30 \mathrm{sec}$. The PCR reaction was normalized to the reference gene: GAPDH. The relative level of each gene was calculated and normalized using the $2^{-\Delta \Delta \mathrm{Cq}}$ formula (22) Each experiment was repeated in triplicate.

Short hairpin (sh)RNA infection. The specific shRNA against HNF1A-AS1 (shHNF1A-AS1) and the scrambled negative control shRNA (shNC) were chemically designed and synthesized by GenePharma Co., Ltd. (Shanghai, China). Lipofectamine ${ }^{\circledR} 2000$ was purchased from Invitrogen; Thermo Fisher Scientific, Inc., and used according to the manufacturer's protocol. The transfection efficiency of the specific shRNA against HNF1A-AS1 in T24 and 5637 cell lines was confirmed by qPCR, as aforementioned.

Cell viability assay. Cell viability was determined using an MTT assay. Briefly, $1 \times 10^{4}$ T24 and 5637 cells were seeded in 96-well plates and transfected with HNF1A-AS1 shRNA (shHNF1A-AS1 group) or scrambled shRNA (shNC group) for $48 \mathrm{~h}$. Cells that were transfected with no shRNA were synchronously co-cultured as a control group. Following treatment, $10 \mu \mathrm{l}$ MTT $(5 \mu \mathrm{g} / \mathrm{ml})$ was mixed with the medium in each well and cells were incubated for an additional $3 \mathrm{~h}$ at $37^{\circ} \mathrm{C}$ in the dark. The formazan crystals that formed were dissolved in $100 \mu \mathrm{l}$ dimethylsulfoxide and the absorbance of
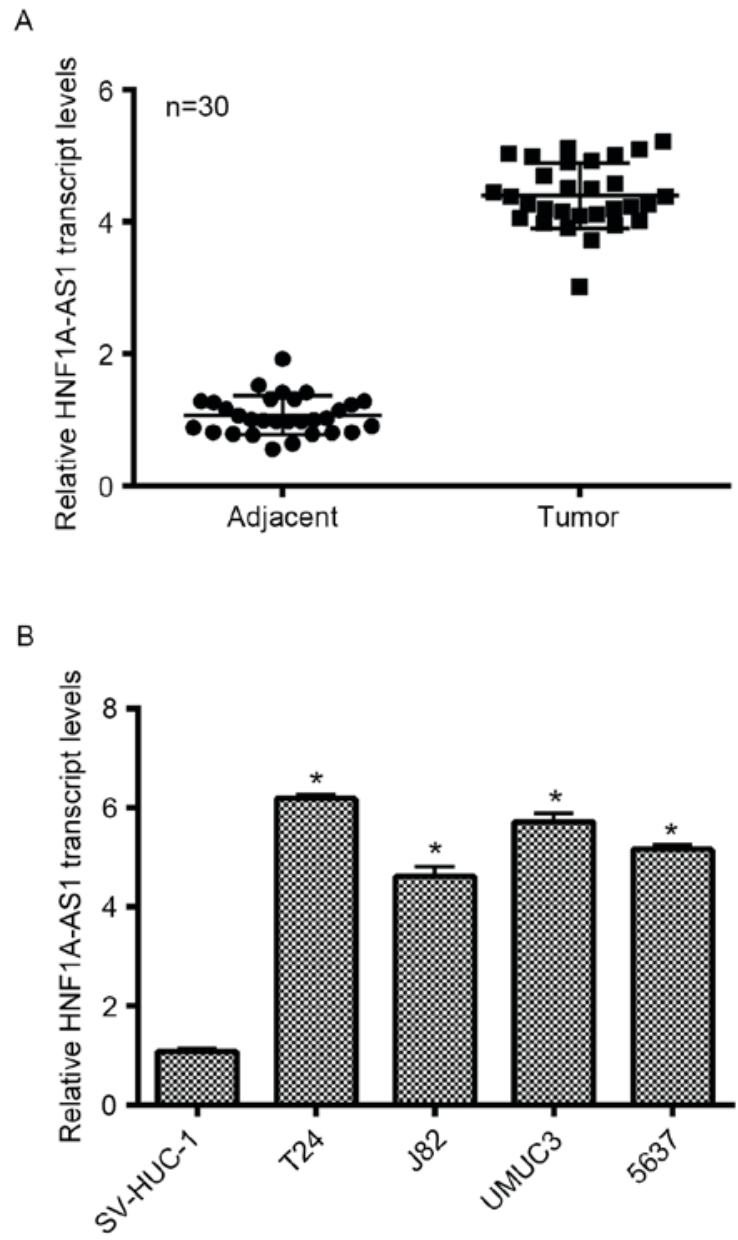

Figure 1. HNF1A-AS1 is upregulated in clinical BC tissues and cultured BC cells. (A) qPCR analysis of the 30 clinical patients with BC and their adjacent non-cancerous tissues. The relative transcript levels of HNF1A-AS1 in BC tissues were increased $~ 4$-fold compared with that of adjacent tissues. (B) qPCR analysis of the transcript level of HNF1A-AS1 in four BC cell lines and one normal urothelial SV-HUC-1 cell line. The relative transcript levels in the four BC cell lines were significantly increased compared with the control. "P<0.05 vs. SV-HUC-1. HNF1A-AS1, hepatocyte nuclear factor 1A-antisense RNA1; BC, bladder cancer; qPCR, quantitative polymerase chain reaction.

each well of the 96-well plate was measured at $570 \mathrm{nM}$ with a plate reader (Tecan Schweiz AG, Mannedorf, Switzerland).

Colony formation assay. A total of $10 \times 10^{3} \mathrm{~T} 24$ and 5637 cells were seeded into 6-well plates and treated with specific shRNA against HNF1A-AS1 (shHNF1A-AS1 group) or scrambled shRNA (shNC group). Control cells were also synchronously cultured (control group). Following treatment for $24 \mathrm{~h}$, the cell lines were suspended and re-seeded into 12-well plates (100 cells/well) in triplicate. The cultured medium was replaced every other day. Following incubation for 10 days, the colonies from three groups were fixed with ice-cold methanol and stained with $1 \%$ crystal violet. A colony was considered to be present when the number of assembled cells was $>50$. Subsequently, the number of colonies was determined under a light microscope at $\mathrm{x} 200$ magnification (Nikon Corporation, Tokyo, Japan). The following formula was used to calculate the rate of colony formation: Colony formation rate $=$ (number of colonies/number of seeded cells) x $100 \%$. 
A

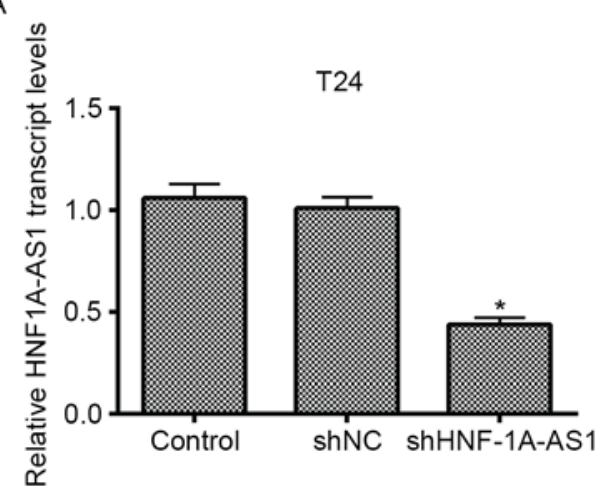

C

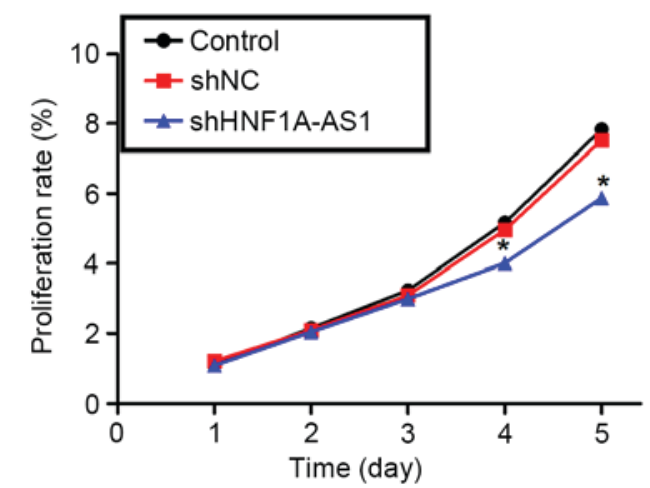

E

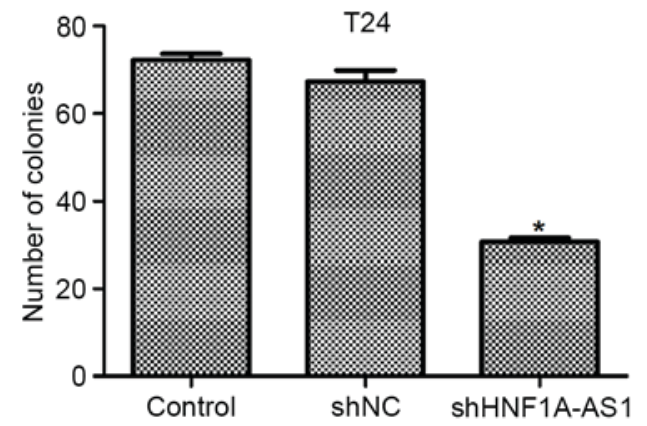

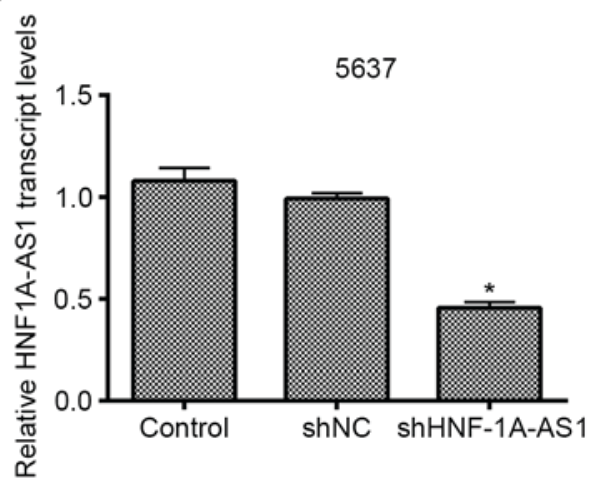

D

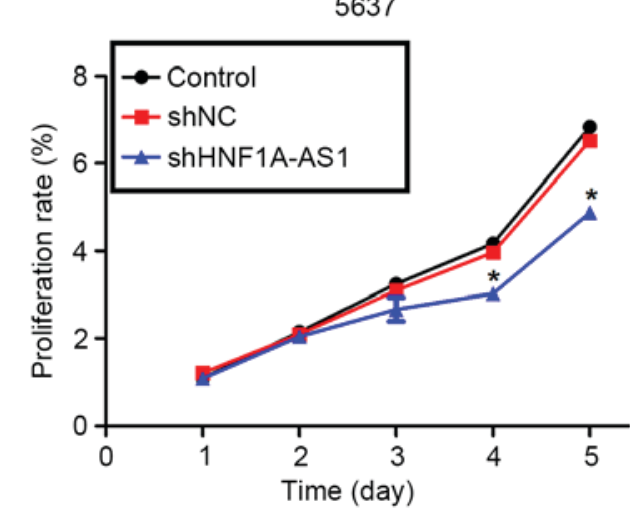

F

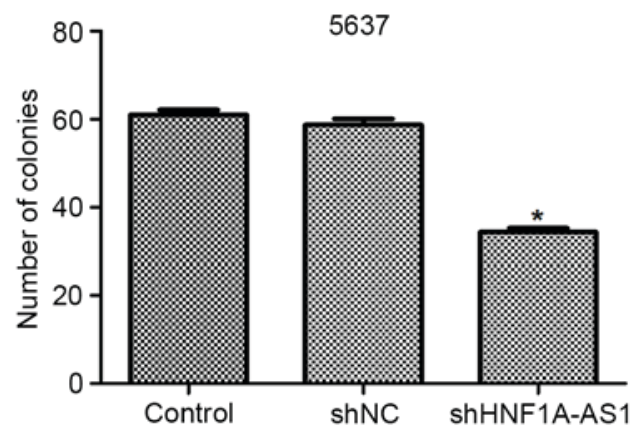

Figure 2. Knockdown of HNF1A-AS1 decreases cell viability and colony formation ability. (A) T24 cells were infected with shRNA and subjected to qPCR analysis to examine the relative transcript level of HNF1A-AS1.(B) 5637 cells were infected with specific shRNA against HNF1A-AS1 and subjected to qPCR analysis to examine the relative transcript level. ${ }^{*} \mathrm{P}<0.05$ vs. control. Knockdown of HNF1A-AS1 decreased the cell viability rate in (C) T24 and (D) 5637 cells on the 4th and 5th day. "P<0.05 vs. control. (E) T24 and (F) 5637 cells were transfected with HNF1A-AS1 shRNA prior to assays, and then subjected to colony formation assays. Data from T24 and 5637 cells demonstrated that the knockdown of HNF1A-AS1 decreased the number of colonies that were formed. ${ }^{*} \mathrm{P}<0.05$ vs. control. HNF1A-AS1, hepatocyte nuclear factor 1A-antisense RNA1; qPCR, quantitative polymerase chain reaction.

Transwell assay. A total of $5 \times 10^{3} \mathrm{~T} 24$ and 5637 cells were seeded into 12-well plates and transfected with specific shRNA or scrambled shRNA for $48 \mathrm{~h}$. Subsequently, cells were harvested and diluted to a concentration of $5 \times 10^{5} / \mathrm{ml}$ with the DMEM without FBS. For the migration assay, a $100 \mu \mathrm{l}$ aliquot of cells was added into the upper chambers (Corning Incorporated, Corning, NY, USA) and $200 \mu 1$ medium with $10 \%$ FBS was added to the lower chamber. After $8 \mathrm{~h}$ of incubation at $37^{\circ} \mathrm{C}$, cells were washed with PBS three times, fixed with methanol and stained with $1 \%$ crystal violet for $5 \mathrm{~min}$. For the invasion assay, the Transwell chambers were coated with Matrigel (BD Biosciences, San Jose, CA, USA) $6 \mathrm{~h}$ before the experiment at $37^{\circ} \mathrm{C}$ in an incubator. Images of the cells on the lower surface of the chamber were captured with a light microscope, and counted in six random fields. All experiments were repeated at least three times in triplicate.

Cell cycle assay. A total of $5 \times 10^{3} \mathrm{~T} 24$ and 5637 cells were seeded into 12-well plates and transfected with control and specific shRNAs. After $48 \mathrm{~h}$ of incubation, cells were harvested and fixed with $75 \%$ ice-cold ethanol overnight at $4^{\circ} \mathrm{C}$. Subsequently, cells were washed with PBS twice and stained with $50 \mathrm{mg} / \mathrm{ml}$ propidium iodide supplied with $50 \mathrm{mg} / \mathrm{ml}$ RNase A (DNase-free) at $37^{\circ} \mathrm{C}$ for an additional $30 \mathrm{~min}$. The percentage of cells in each phase in the two cell lines was calculated using flow cytometry. The experiments were repeated three times with each test in triplicate. 
Statistical analysis. All data are presented as the mean \pm standard deviation. Each experiment was repeated at least three times in triplicate. A two-tailed Student's t-test was used to compare means of two groups, whereas one-way analysis of variance was used for comparisons among multiple groups ( $\geq 3$ groups), followed by a least significant difference post hoc test. $\mathrm{P}<0.05$ was considered to indicate a statistically significant difference.

\section{Results}

HNF1A-AS1 is upregulated in clinical BC tissues and cultured $B C$ cells. Initially, the expression of lncRNA HNF1A-AS1 was detected in patients with $\mathrm{BC}$ and cultured BC cell lines. As no antibody is currently available for HNF1A-AS1, only the transcript level was detected in the present study. As presented in Fig. 1A, the transcript levels of HNF1A-AS1 in 30 clinical $\mathrm{BC}$ tissues extracted from patients were significantly increased (>4-fold) compared with the adjacent non-cancerous tissues. SV-HUC-1 cells are normal human urothelial cells that are used as control cells. This cell line was cultured as an internal control in the present study. Simultaneously, four other BC cell lines (T24, J82, UMUC3 and 5637) were synchronously cultured. The relative expression of HNF1A-AS1 in different $\mathrm{BC}$ cell lines was examined, and it was demonstrated that the transcript levels of HNF1A-AS1 in the entire BC cell lines were notably upregulated compared with that in SV-HUC-1 cells. Remarkably, the transcript level of HNF1A-AS1 was increased 6.2-fold in T24 cells and 5.8-fold in UMUC3 cells compared with the control cells. Furthermore, the transcript level of HNF1A-AS1 was increased 4.6-fold in J82 cells and 5.1-fold in 5637 cells. T24 and 5637 cells were selected as representative $\mathrm{BC}$ cells for subsequent analyses. These results revealed that HNF1A-AS is significantly overexpressed in clinical $\mathrm{BC}$ tissues and $\mathrm{BC}$ cells cultured in vitro.

Knockdown of HNF1A-AS1 is successfully achieved by specific shRNA transfection. To elucidate the detailed role of HNF1A-AS1 in human BC, specific shRNA against HNF1A-AS1 (shHNF1A-AS1) was designed and synthesized. Transfection of scrambled shRNA (shNC) did not markedly change the transcript level of HNF1A-AS1 in the T24 and 5637 cell lines. However, when the cells were treated with the specific shRNA, the transcript level of HNF1A-AS1 was significantly decreased ( $\sim 50 \%)$ in the two cell lines (Fig. 2A and $\mathrm{B}$ ). These results indicated the high efficiency of specific shRNA to deplete HNF1A-AS1.

Knockdown of HNF1A-AS1 inhibits cell viability and colony formation in T24 and 5637 cells. The role of HNF1A-AS in human $\mathrm{BC}$ was investigated using a cell viability assay. No significant differences in cell viability among the three groups in the first 3 days for the T24 and 5637 cells were identified (Fig. 2C and D). However, the viability of the T24 cells was suppressed by $27 \%$ on the fourth day and by $30 \%$ on the fifth day (Fig. 2C). For the 5637 cells, the viability was decreased by $27 \%$ on the fourth day and the inhibition of 5637 and $35 \%$ on the fifth day (Fig. 2D). The colony formation assay additionally demonstrated that the knockdown of HNF1A-AS by specific shRNA remarkably decreased the numbers of colonies
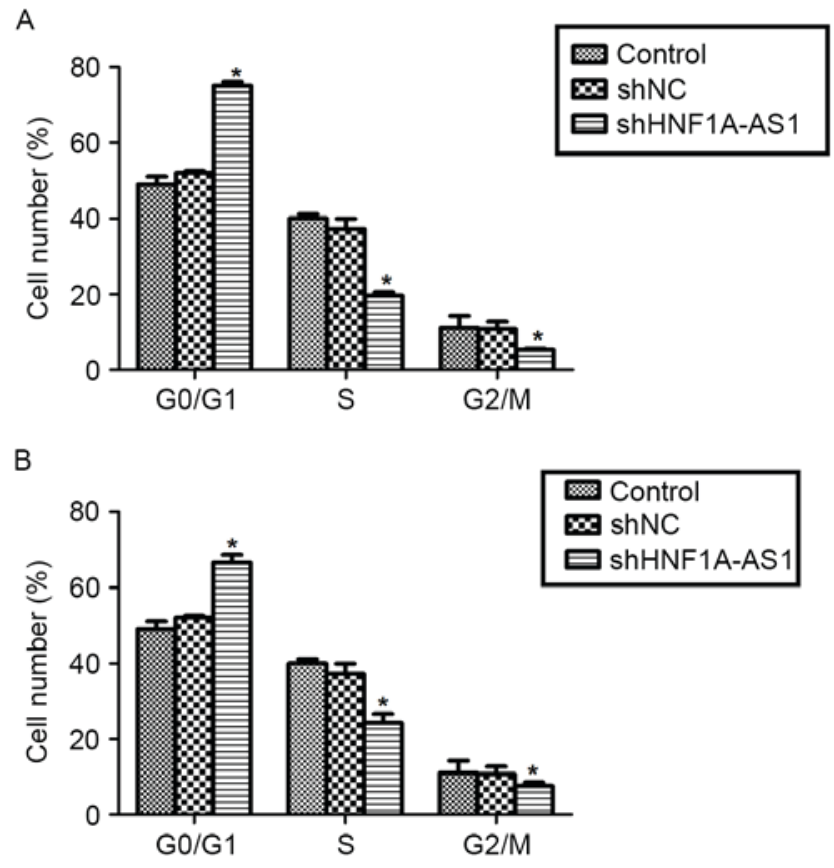

Figure 3. Knockdown of HNF1A-AS1 arrests the cell cycle in $G_{0} / G_{1}$ phase in T24 and 5637 cells. (A) T24 cells were transfected with a specific shRNA against HNF1A-AS1 and subjected to flow cytometric analysis. The percentage of $\mathrm{T} 24$ cells in each stage was determined. ${ }^{*} \mathrm{P}<0.05$ vs. control. (B) 5637 cells were infected with specific shRNA, fixed by ethanol and used for flow cytometric assays. The percentage of 5637 cells in each stage was determined. "P $<0.05$ vs. control. HNF1A-AS1, hepatocyte nuclear factor 1A-antisense RNA1; shRNA/sh, short hairpin RNA; NC, negative control.

for the two BC cell lines (Fig. 2E and F). Quantification of the number of colonies for T24 cells revealed that $\sim 70$ colonies were formed in control shRNA-treated cells, whereas only 34 colonies were observed in shHNF1A-AS1-transfected cells. Similarly, in 5637 cells, the number of colonies formed on agar plates was also decreased by $42 \%$ in specific shRNA-treated cells compared with the control cells. These results indicate that HNF1A-AS1 promotes cell viability and colony formation in the $\mathrm{BC}$ cell lines.

Knockdown of HNF1A-AS1 arrests cell cycle in $G_{0} / G_{1}$ phase in T24 and 5637 cells. To explain the inhibitive effects by shHNF1A-AS1 on viability, the cell cycle distribution in T24 and 5637 cells was analyzed using flow cytometry. The knockdown of HNF1A-AS1 in T24 cells increased the proportion of cells in $\mathrm{G}_{0} / \mathrm{G}_{1}$ phase by $60 \%$, whereas it decreased the proportion of cells in $S$ phase by $50 \%$ and that in $\mathrm{G}_{2} / \mathrm{M}$ phase by $48 \%$ (Fig. 3A). Similarly, the knockdo wn of HNF1A-AS1 in 5637 cells increased the proportion of cells in $\mathrm{G}_{0} / \mathrm{G}_{1}$ phase by $36 \%$, whereas it decreased the proportion of cells in $S$ phase and $\mathrm{G}_{2} / \mathrm{M}$ phase by 40 and $31 \%$, respectively (Fig. 3B). These results indicate that the knockdown of HNF1A-AS1 arrested $\mathrm{BC}$ cell cycle progression by notably accumulating cells in $\mathrm{G}_{0} / \mathrm{G}_{1}$ phase in $\mathrm{T} 24$ and 5637 cells.

Knockdown of HNF1A-AS1 inhibits BC cell migration and invasion in vitro. The effects of HNF1A-AS1 knockdown on cell migration and invasion in vitro were investigated. Numerous T24 and 5637 cells migrated through 

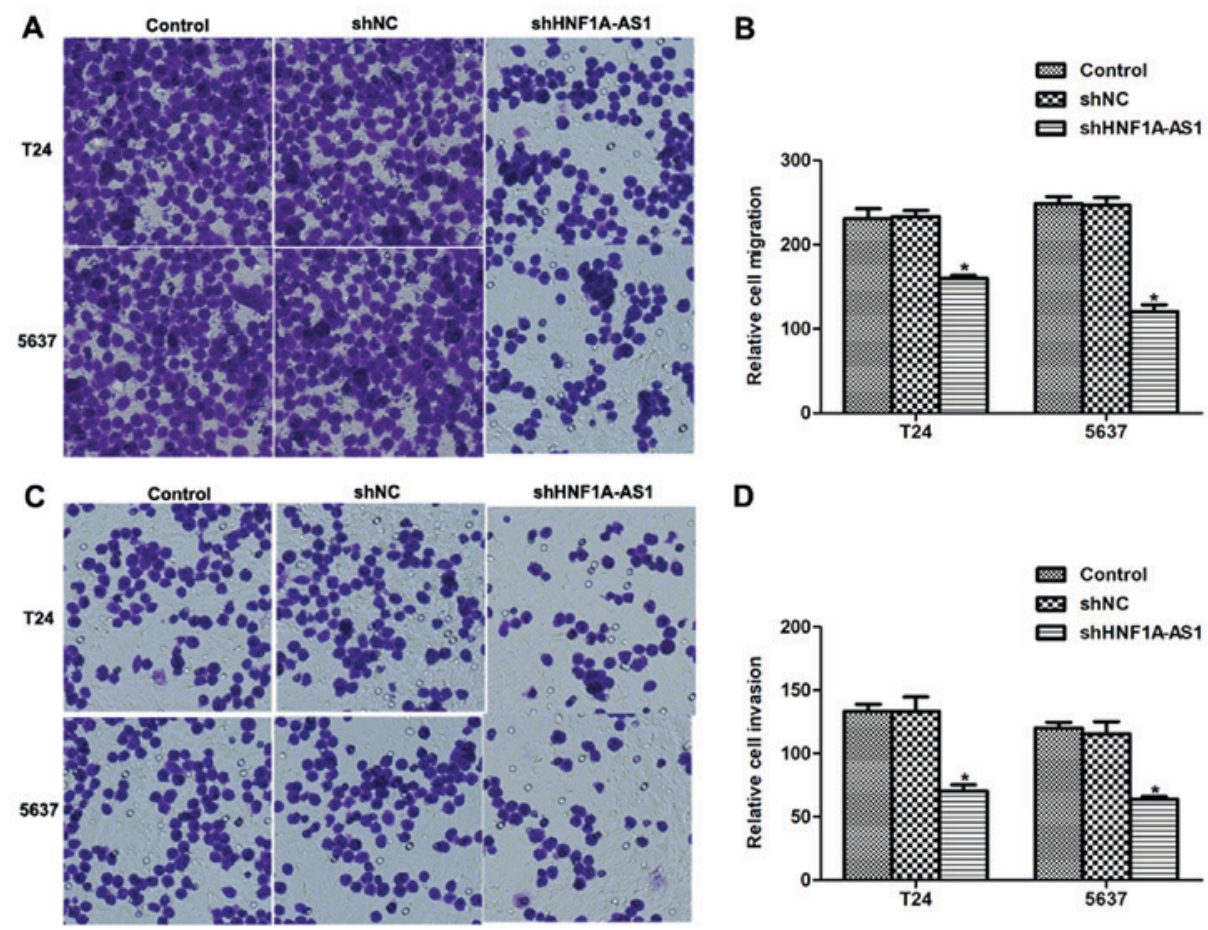

Figure 4. HNF1A-AS1 promotes BC cell migration and invasion in vitro. (A) Representative images of cell migration experiment for T24 and 5637 BC cells. The number of migrated cells decreased visually when cells were depleted using HNF1A-AS1. (B) Migrated cells on the lower surface of the chamber were stained with $1 \%$ crystal violet and counted. The knockdown of HNF1A-AS1 in T24 and 5637 cells decreased the number of migrated cells. ${ }^{*}<0.05$ vs. control. (C) Representative images of cell invasion assay for BC cell lines following transfection with the specific shRNA against HNF1A-AS1. (D) Quantification of invaded cells on the lower surface of the chamber for the invasion assays. The knockdown of HNF1A-AS1 decreased the cell invasion to 50\% compared with that in the control group. " P<0.05 vs. control. HNF1A-AS1, hepatocyte nuclear factor 1A-antisense RNA1; BC, bladder cancer; sh, short hairpin RNA; $\mathrm{NC}$, negative control.

the membrane (Fig. 4A and B); however, notable disparities between the control and HNF1A-AS1 shRNA-treated groups were observed. For the migration assays, the number of cells migrating to the lower surface of the chamber was decreased by $31 \%$ for T 24 cells and $44 \%$ for 5637 cells following knockdown of HNF1A-AS1 (Fig. 4B). For the invasion assays, the differences between specific shRNA-treated and control groups were $45 \%$ for T24 cells and $48 \%$ for 5637 cells (Fig. 4C and D). These results suggest that HNF1A-AS1 may promote T24 and 5637 cell metastases in vitro.

\section{Discussion}

$\mathrm{BC}$ is one of the most common types of cancer, with $>12$ million incident diagnoses and $>1.3$ million mortalities each year (23). BC may be categorized into non-muscle-invasive BC (80\%) and muscle-invasive BC (20\%) on the basis of the scale of tumor infiltration into the musculature of the bladder of the patient (24). Non-muscle-invasive BC exhibits a high tendency for recurrence, which leads to its high prevalence in the USA (24). In addition, between 10 and $30 \%$ of non-muscle-invasive $\mathrm{BC}$ progresses to the muscle-invasive phenotype, which results in a markedly decreased 5-year survival rate (25). Therefore, the early detection of $\mathrm{BC}$ is a priority for improving the outcomes of patients with BC.

In the present study, the role of the previously seldom studied but widely expressed lncRNA HNF1A-AS1 was explored. First, it was identified that HNFA-AS1 was upregulated in clinical BC tissues and cultured BC cells.
The knockdown of HNF1A-AS1 resulted in a decreased cell viability rate in vitro. Concurrently, it was observed that the cell cycle was arrested in $G_{0} / G_{1}$ phase, indicating that the inhibitory effects on viability by the knockdown of HNF1A-AS1 may be achieved through interrupting cell cycle progression. Additionally, the knockdown of HNF1A-AS1 inhibited cell migratory and invasive abilities in the two cell lines used in the present study. To the best of our knowledge, the present study is the first to identify the role of HNF1A-AS1 in BC. Taken together, these results suggest that HNF1A-AS1 serves critical roles in $\mathrm{BC}$ cell viability and migration. The dysregulation of HNF1A-AS1 in BC may contribute to the development and progression of $\mathrm{BC}$.

HNF1A-AS1 was indicated to be associated with the progression of lung adenocarcinoma, esophageal adenocarcinoma $(20,21)$ and, most recently, in gastric cancer (26). Functionally, HNF1A-AS1 is consistently recognized as a regulator of cell viability and metastasis in the aforementioned types of cancer. However, no detailed mechanisms underlying its biological activity have been revealed. Previously, Yang et al (20) have demonstrated that HNF1A-AS1 was involved in the regulation of lncRNA H19, another widely studied cancer-associated lncRNA. Concurrently, it was indicated that HNF1A-AS1 knockdown preferentially affected genes that are linked to assembly of chromatin and the nucleosome, a mechanism essential to cell cycle progression (15). In that study, the well-known cancer-associated lncRNA H19 was the gene most markedly inhibited by HNF1A-AS1 knockdown. These results suggest that lncRNA H19 may elicit 
its effects through HNF1A-AS1. H19 may be a critical downstream effector that transduces signals from HNF1A-AS1, and therefore may contribute to HNF1A-AS1-mediated cell viability and metastasis, particularly in the regulation of cell cycle progression. Therefore, it would be interesting to investigate the association between HNF1A-AS1 and H19 in BC in additional studies.

In conclusion, the present study suggests that HNF1A-AS1 was overexpressed in BC. The knockdown of HNF1A-AS1 inhibited cell viability and metastasis in BC cells. These results may lead to the identification of novel methods to diagnose and treat $\mathrm{BC}$ in clinical settings.

\section{References}

1. Jemal A, Bray F, Center MM, Ferlay J, Ward E and Forman D: Global cancer statistics. CA Cancer J Clin 61: 69-90, 2011.

2. Sylvester RJ, van der Meijden AP, Oosterlinck W, Witjes JA, Bouffioux C, Denis L, Newling DW and Kurth K: Predicting recurrence and progression in individual patients with stage Ta T1 bladder cancer using EORTC risk tables: A combined analysis of 2596 patients from seven EORTC trials. Eur Urol 49: 466-477, 2006

3. Kurth KH, Denis L, Bouffioux C, Sylvester R, Debruyne FM, Pavone-Macaluso $\mathrm{M}$ and Oosterlinck W: Factors affecting recurrence and progression in superficial bladder tumours. Eur J Cancer 31A: 1840-1846, 1995.

4. Fernandez-Gomez J, Madero R, Solsona E, Unda M, Martinez-Piñeiro L, Gonzalez M, Portillo J, Ojea A, Pertusa C, Rodriguez-Molina $\mathrm{J}$, et al: Predicting nonmuscle invasive bladder cancer recurrence and progression in patients treated with bacillus Calmette-Guerin: The CUETO scoring model J Urol 182: 2195-2203, 2009.

5. Zhu H, Li X, Song Y, Zhang P, Xiao Y and Xing Y: Long non-coding RNA ANRIL is up-regulated in bladder cancer and regulates bladder cancer cell proliferation and apoptosis through the intrinsic pathway. Biochem Biophys Res Commun 467: 223-228, 2015

6. Dancik GM, Owens CR, Iczkowski KA and Theodorescu D: A cell of origin gene signature indicates human bladder cancer has distinct cellular progenitors. Stem Cells 32: 974-982, 2014

7. Peter S, Borkowska E, Drayton RM, Rakhit CP, Noon A, Chen W and Catto JW: Identification of differentially expressed long noncoding RNAs in bladder cancer. Clin Cancer Res 20: 5311-5321, 2014 .

8. Zhang Q, Su M, Lu G and Wang J: The complexity of bladder cancer: Long noncoding RNAs are on the stage. Mol Cancer 12: $101,2013$.

9. Sun X, Du P, Yuan W, Du Z, Yu M, Yu X and Hu T: Long non-coding RNA HOTAIR regulates cyclin J via inhibition of microRNA-205 expression in bladder cancer. Cell Death Dis 6: e1907, 2015.

10. Zhuang C, Li J, Liu Y, Chen M, Yuan J, Fu X, Zhan Y, Liu L, Lin J, Zhou Q, et al: Tetracycline-inducible shRNA targeting long non-coding RNA PVT1 inhibits cell growth and induces apoptosis in bladder cancer cells. Oncotarget 6: 41194-41203, 2015.
11. Martin L and Chang HY: Uncovering the role of genomic 'dark matter' in human disease. J Clin Invest 122: 1589-1595, 2012.

12. Wang Y, He L, Du Y, Zhu P, Huang G, Luo J, Yan X, Ye B, $\mathrm{Li} \mathrm{C}$, Xia P, et al: The long noncoding RNA lncTCF7 promotes self-renewal of human liver cancer stem cells through activation of Wnt signaling. Cell Stem Cell 16: 413-425, 2015.

13. Han X, Yang F, Cao H and Liang Z: Malat1 regulates serum response factor through miR-133 as a competing endogenous RNA in myogenesis. FASEB J 29: 3054-3064, 2015.

14. Li P, Ruan X, Yang L, Kiesewetter K, Zhao Y, Luo H, Chen Y, Gucek M, Zhu J and Cao H: A liver-enriched long non-coding RNA, lncLSTR, regulates systemic lipid metabolism in mice. Cell Metab 21: 455-467, 2015.

15. Han P, Li W, Lin CH, Yang J, Shang C, Nuernberg ST, Jin KK, $\mathrm{Xu} W$, Lin CY, Lin CJ, et al: A long noncoding RNA protects the heart from pathological hypertrophy. Nature 514: 102-106, 2014.

16. Panzitt K, Tschernatsch MM, Guelly C, Moustafa T, Stradner M, Strohmaier HM, Buck CR, Denk H, Schroeder R, Trauner M and Zatloukal K: Characterization of HULC, a novel gene with striking up-regulation in hepatocellular carcinoma, as noncoding RNA. Gastroenterology 132: 330-342, 2007.

17. Ji P, Diederichs S, Wang W, Böing S, Metzger R, Schneider PM, Tidow N, Brandt B, Buerger H, Bulk E, et al: MALAT-1, a novel noncoding RNA, and thymosin beta4 predict metastasis and survival in early-stage non-small cell lung cancer. Oncogene 22: 8031-8041, 2003

18. Wu W, Zhang S, Li X, Xue M, Cao S and Chen W: Ets-2 regulates cell apoptosis via the Akt pathway, through the regulation of urothelial cancer associated 1, a long non-coding RNA, in bladder cancer cells. PLoS One 8: e73920, 2013.

19. Zhuang J, Lu Q, Shen B, Huang X, Shen L, Zheng X, Huang R, Yan J and Guo H: TGF $\beta 1$ secreted by cancer-associated fibroblasts induces epithelial-mesenchymal transition of bladder cancer cells through lncRNA-ZEB2NAT. Sci Rep 5: 11924, 2015.

20. Yang X, Song JH, Cheng Y, Wu W, Bhagat T, Yu Y, Abraham JM, Ibrahim S, Ravich W, Roland BC, et al: Long non-coding RNA HNF1A-AS1 regulates proliferation and migration in oesophageal adenocarcinoma cells. Gut 63: 881-890, 2014.

21. Wu Y, Liu H, Shi X, Yao Y, Yang W and Song Y: The long non-coding RNA HNF1A-AS1 regulates proliferation and metastasis in lung adenocarcinoma. Oncotarget 6: 9160-9172, 2015.

22. Livak KJ and Schmittgen TD: Analysis of relative gene expression data using real-time quantitative PCR and the 2(-Delta Delta C(T)) method. Methods 25: 402-408, 2001.

23. Ploeg M, Aben KK and Kiemeney LA: The present and future burden of urinary bladder cancer in the world. World J Urol 27: 289-293, 2009.

24. Millán-Rodríguez F, Chéchile-Toniolo G, Salvador-Bayarri J, Palou J, Algaba F and Vicente-Rodríguez J: Primary superficial bladder cancer risk groups according to progression, mortality and recurrence. J Urol 164: 680-684, 2000.

25. Rübben H, Lutzeyer W, Fischer N, Deutz F, Lagrange W and Giani G: Natural history and treatment of low and high risk superficial bladder tumors. J Urol 139: 283-285, 1988.

26. Dang Y, Lan F, Ouyang X, Wang K, Lin Y, Yu Y, Wang L, Wang $\mathrm{Y}$ and Huang Q: Expression and clinical significance of long non-coding RNA HNF1A-AS1 in human gastric cancer. World J Surg Oncol 13: 302, 2015. 